Space Weather

\section{RESEARCH ARTICLE \\ 10.1002/2014SW001113 \\ Probabilistic forecasting analysis of geomagnetic indices for southward IMF events}

Key Points:

- SW/IMF shows significant difference before strong IMF $B_{s}$ for different transients

- SW density weakly affects magnetosphere, depending on the type of transient

- ULF waves are induced by strong IMF $B_{S}$ and SW dynamic pressure disturbances

Correspondence to:

X.-Y. Zhang,

zhangxy@umich.edu

\section{Citation:}

Zhang, X.-Y., and M. B. Moldwin (2015), Probabilistic forecasting analysis of geomagnetic indices for southward IMF events, Space Weather, 13, 130-140, doi:10.1002/2014SW001113.

Received 22 AUG 2014 Accepted 21 JAN 2015 Accepted article online 30 JAN 2015 Published online 4 MAR 2015

\author{
X.-Y. Zhang' and M. B. Moldwin' ${ }^{1}$ \\ ${ }^{1}$ Department of Atmospheric, Oceanic, and Space Sciences, University of Michigan, Ann Arbor, Michigan, USA
} events with in situ solar wind data.
Abstract Geomagnetic disturbances that drive space weather impacts such as ground-induced currents and radiation belt enhancements are usually driven by strong southward interplanetary magnetic field (IMF) intervals. However, current heliospheric models either do not predict or provide low-accuracy forecasts of IMF $B_{z}$. Here we examine the probability distribution function of geomagnetic activity indices for southward IMF intervals. We analyze the in situ plasma and magnetic field measurements long-duration large-amplitude southward IMF intervals (called $B_{s}$ events). The statistical profiles of other solar wind and IMF parameters show significant differences during the periods 1 day before the $B_{s}$ events for different solar wind transients (such as interplanetary coronal mass ejections and stream interaction regions). As is well known, we find that the solar wind speed is positively correlated with geomagnetic indices and that strong southward IMF is the key in storm triggering but not necessarily for substorms. We find that the solar wind density weakly affects geomagnetic field activity, but the response depends on the type of solar wind transient that includes the strong $B_{s}$ events. We also find that magnetospheric ultralow-frequency waves are induced by both strong southward IMF and solar wind dynamic pressure disturbances. We suggest that strong $B_{s}$ events could be predicted from the preceding characteristics of solar wind and IMF changes and that probabilistic forecasting of geomagnetic activity occurrence is potentially useful in space weather forecasting. We present preliminary analysis to demonstrate the out-of-sample ability to predict IMF $B_{s}$

\section{Introduction}

The structure of solar wind and the orientation of the interplanetary magnetic field (IMF) determine the level of geomagnetic activity, which is characterized by dramatic changes in the plasmasphere, radiation belts, and the ionosphere. These changes impact the normal use of satellites, GPS signals, and even the electric power system. Extensive studies have shown that the southward component of IMF (southward IMF) is the predominant factor of the upstream driver. However, current heliospheric models do not predict or provide low-accuracy prediction of this parameter [Jin et al., 2012; Liou et al., 2014; Shen et al., 2014]. Thus, the development of methods to provide accurate prediction of southward IMF in the near-Earth region is essential for the development of space weather models.

Based on in situ observations at $1 \mathrm{AU}$, previous studies have defined several solar wind transients that are potential triggers of geomagnetic disturbances, such as interplanetary coronal mass ejections (ICMEs), stream interaction regions (SIRs), and so on [Denton et al., 2006; Tsurutani et al., 2006, 2013]. The different solar wind transients are identified by their different plasma and magnetic field signatures. Zurbuchen and Richardson [2006] summarized the observational signatures of magnetic cloud ICMEs, which are an enhancement ( $>10 \mathrm{nT}$ ) of magnetic field amplitude, along with smooth rotation of its polarity, and also a decreasing velocity/density profile. Jian et al. [2006a] did a statistical study of SIRs during 1995-2004 and concluded that the averaged peak pressure of an SIR is $176 \pm 6 \mathrm{pPa}$, and averaged speed increase is $230 \pm 5$ km/s. In our previous studies [Zhang and Moldwin, 2014; Zhang et al., 2014], we identified long-duration and large-amplitude IMF $B_{z}$ south intervals and categorized them based on the type of solar wind transient they were associated with.

The geoeffectiveness of different kinds of solar wind structures with various combinations of plasma and magnetic field conditions has been analyzed widely using both observations and simulations. Denton et al. [2006] performed a statistical study of the magnetospheric plasma's response to ICME and corotating interaction region (CIR) during storm time and proposed that CIR events change the plasma sheet temperature more significantly while ICMEs modulate the density to a greater extent, which is partly 
attributed to the differences in their solar wind speed and density. Echer and Gonzalez [2004] suggested that compound interplanetary magnetic structures are more geoeffective than single interplanetary magnetic structures based on a statistical study of solar wind structures like magnetic clouds, heliospheric current sheet sector boundary crossings, and the Dst index following those intervals. O'Brien et al. [2001] did a statistical study to compare storms with and without the occurrence of relativistic electrons at geosynchronous orbit. They found that solar wind with continuous speed greater than $450 \mathrm{~km} / \mathrm{s}$ and long-lasting, elevated ULF Pc5 wave power in the magnetosphere during the recovery phase of storms are both strongly associated with the appearance of these electrons. Newell et al. [2007] have studied the correlation between 10 different geomagnetospheric indices and 20 candidate solar wind coupling functions using multiple years of data. They found that the function $d \phi_{\mathrm{MP}} / \mathrm{d} t=v^{4 / 3} B_{T}^{3 / 2} \sin ^{8 / 3}\left(\theta_{c} / 2\right)$, where $d \phi_{\mathrm{MP}} / \mathrm{d} t$ is the rate magnetic flux is opened at the magnetopause, $v$ is the solar wind speed, $B_{T}$ is the IMF magnitude, and $\theta_{c}$ is the IMF clock angle, defined by $\theta_{c}=\arctan \left(B_{y} / B_{z}\right)$, is correlated best with all the indices, except Dst. Note that $B_{z}$ south comes into play through the IMF clock angle $\theta_{c}$. Though the conclusion of Newell et al. [2007] is that $B_{z}$ alone only accounts for a little better than a quarter of the variance, IMF $B_{z}$ south is essential in all the coupling functions examined, and hence, prediction of IMF $B_{z}$ south is necessary for forecasting.

The response of magnetospheric ultralow-frequency (ULF) waves to nonstorm-exclusive intervals has also been studied extensively. Takahashi and Ukhorskiy [2008] performed correlation analysis between the solar wind parameters and amplitude of Pc5 waves $(1.7-6.7 \mathrm{mHz}$ ) at geosynchronous orbit for solar minimum year 2006 and concluded that the major driver of geosynchronous Pc5 waves is solar wind pressure variations rather than the Kelvin-Helmholtz instability on the magnetopause. The statistical studies by Sanny et al. [2002, 2007] suggested that solar wind dynamic pressure and its variability have greater influence on ULF wave power than IMF northward/southward orientation and that ULF wave power changes more for northward IMF than for southward IMF during periods of high solar wind dynamic pressure. However, Skoug et al. [2004] pointed out that the solar wind dynamic pressure coupled with large negative $B_{z}$ was sufficient to cause intense geomagnetic disturbances.

The relationship between geomagnetic activity at high-latitude and solar wind/IMF conditions has also been studied for a long time. Tsurutani and Gonzalez [1987] showed that the high-intensity (AE > $1000 \mathrm{nT})$, long-duration ( $T>2$ days) continuous auroral activity events are induced by the southward component of IMF embedded in interplanetary Alfvén wave trains propagating outward from the Sun.

Global simulations have also been used extensively to understand the solar wind drivers of geomagnetic activity. Lopez et al. [2004] demonstrated that solar wind density plays a significant role in modulating the transfer of energy to the magnetosphere under southward IMF using global magnetohydrodynamic (MHD) simulations of the solar wind-magnetosphere interaction. Palmroth et al. [2003] used a 3-D global MHD simulation code to examine the energy flow from the solar wind to the magnetosphere during a magnetic storm and found that the correlation between the simulated total transferred energy and the empirical energy parameter calculated from the solar wind parameters (magnetic field, solar wind speed, and magnetic field clock angle) depends on the phase during the storm. Tóth et al. [2007] carried out the first Sun-to-thermosphere simulation of the most powerful solar eruptions associated with the "Halloween Storms," using observed solar magnetograms, and pointed out that the simulated magnetospheric activity index is in good agreement with the Dst index. Owens et al. [2014] used downscaling input and tested an ensemble of simulation results for the space weather forecasting model, the Lyon-Fedder-Mobbary simulation, and found that the best estimate of magnetospheric forecast is improved and uncertainty is quantifiable. They also suggested that the distribution of solar wind parameter fluctuations should be analyzed for different solar wind types, respectively, and that the coupling of different components should be considered.

McPherron and Siscoe [2004] applied the concept of air mass climatology to solar wind for probabilistic forecasting of geomagnetic indices. They used the stream interface (the interface within a corotating compression region) as "marker," which is identified by a bipolar deflection of solar wind flow in the east-west (EW) direction. They set the polarity change of the EW flow deflection as the zero epoch time and carried out superposed epoch analysis of solar wind/IMF parameters and analysis of cumulative probability distribution functions for geomagnetic index ap for corotating interaction regions in 1995. Here we perform a follow-up study of McPherron and Siscoe [2004], focusing on the southward component of 
IMF as a marker. Although many studies have shown the correlation between magnetospheric activity and multiple solar wind/IMF parameters, the southward IMF is still the most important and worst predicted component. Thus, it is important to study the solar wind and IMF behavior as well as geomagnetic activity before and during the southward IMF intervals to learn more about their precursors and effects. We include more types of solar wind transients such as ICMEs, SIRs, and Alfvénic events and also extend the analysis to geomagnetic indices like SYM- $H, A E$, and ULF wave power and nearly a complete solar cycle of ICME- and SIR-related events (1995-2004) for a comprehensive study of solar wind-magnetosphere interaction.

In this study, we use the database of southward IMF events related with different types of solar wind structures [Zhang and Moldwin, 2014; Zhang et al., 2014] and the solar wind, IMF, and geomagnetic activity indices data from in situ and ground observations to derive statistics of solar wind conditions and geomagnetic response during the 2 days around the change of IMF to more southward. The knowledge of the relationship between other solar wind/IMF parameters and southward IMF is important to understand their source on the Sun and their propagation through the interplanetary medium and also key to improve the forecasting ability of the IMF $B_{z}$ component based on simulating the other parameters in current solar/heliospheric models. The knowledge of how different combinations of solar wind conditions affect the Earth's magnetosphere is also important to understand the physical mechanisms that drive geomagnetic activity to improve heliosphere-magnetosphere modeling and to enhance the ability of space weather prediction based on in situ observations.

\section{Methodology}

Zhang and Moldwin [2014] and Zhang et al. [2014] identified strong $B_{s}$ events ( $t>1 \mathrm{~h}$ and $B_{z}<-5 \mathrm{nT}$ ) and associated them with well-defined solar wind structures (ICME, SIR, and Alfvénic events) based on in situ observations and published lists. In this study, we examine the $1 \mathrm{~min}$ magnetic field data and $92 \mathrm{~s}$ plasma data at 1 AU in the period of 1995-2004 (1998-2004 for Alfvénic events) measured by the magnetometer on board the Wind satellite and downloaded from Coordinated Data Analysis Web, in order to study the features of the solar wind/IMF parameters during southward IMF intervals ( $t>1 \mathrm{~h}$ and $B_{z}<-5 \mathrm{nT}$ ) associated with different types of solar wind phenomena. We used the Wind data because the published data sets of the solar wind transients (ICME and SIR) are mostly based on the measurements from Wind, and also, Wind has a larger time span of available observations. However, we use ACE data in the preliminary prediction capability analysis. The symmetric (SYM)- $H$ is the index to describe the geomagnetic disturbance fields in the horizontal direction in midlatitudes with high time resolution (1 $\mathrm{min}$ ). The auroral electrojet $(A E)$ index is derived from geomagnetic variations in the horizontal component observed at selected observatories along the auroral zone in the Northern Hemisphere to represent the overall activity of the electrojets. SYM- $H$ and $A E$ indices are widely used to get a comprehensive understanding of the activity in the magnetosphere, especially the well-known geomagnetic storm and substorm. Thus, we also analyze the geomagnetic activity indices (SYM-H and $A E$ ) obtained from the OMNI data set and magnetic field data at geosynchronous orbit measured by GOES satellites provided by CDAWeb.

Our methodology is as follows:

1. Setting the start time of the southward IMF events ( $t>1 \mathrm{~h}$ and $B_{z}<-5 \mathrm{nT}$ ) related with SIR, ICME, shock, and Alfvénic events identified by Zhang and Moldwin [2014] and Zhang et al. [2014] as the zero epoch time. We then performed a superposed epoch analysis of solar wind/IMF parameters, such as the IMF $B_{z}$, IMF magnitude $\left(B_{t}\right)$, solar wind speed $\left(V_{s w}\right)$, and proton density $\left(N_{p}\right)$, for $24 \mathrm{~h}$ before and $24 \mathrm{~h}$ after the zero epoch time using the $1 \mathrm{~min}$ averaged data. It is worth noting that the zero epoch time is not when the IMF becomes negative, but when it falls below $-5 \mathrm{nT}$. We accept the potential overlaps in intervals in epoch selection process and do not remove other events that could occur within $24 \mathrm{~h}$. The superposed epoch analysis (SEA) technique is a statistical method used to resolve significant signal-to-noise problems. Through simple compositing, the SEA method involves sorting data into categories dependent on a "key time" for synchronization and then comparing the means or medians of those categories. The method allows the analysis of systematic behavior of a parameter with respect to an âŁevent.âŁž In our study, SEA allows the identification of any systematic behavior prior to the start of the IMF $B_{z}$ event. Examples of applications of the SEA method are widespread in various scientific fields of study and are extensively used in space physics [e.g., O'Brien et al., 2001; Green et al., 2004]. 
2. Since a preliminary analysis shows that most features of solar wind/IMF-magnetosphere variations occur in the -12 to $12 \mathrm{~h}$ intervals around the zero epoch time, we extract the $12 \mathrm{~h}$ data before and after the zero epoch time for all the events. We then compare the averaged values of $V_{s w}, E_{y}, N_{p}$, and dynamic pressure $\left(P_{\mathrm{sW}}\right)$ during each of the $12 \mathrm{~h}$ intervals and their median values for all the events. We examined the distribution of the solar wind parameters to understand how best to statistically characterize them. We found that the solar wind speed is Gaussian like and the proton density is a lognormal distribution. Also, our data set covers the southward IMF events for 7 years (Alfvénic events) and 10 years (ICME and SIR events). Thus, the median values are reasonable baselines to distinguish the higher and lower level of the specific parameters. We choose these solar wind parameters since they are considered as important components for geomagnetic activity, and they are the parameters provided by current heliospheric models. These cases are categorized into different classes, such as fast/slow, large/small dynamic pressure variation, or high/low density, by dividing the distribution into two parts above and below the median. We then calculate the cumulative probability of the minimum SYM-H index value during the two $12 \mathrm{~h}$ periods, which gives the probability that the absolute value of minimum SYM- $H$ will exceed the absolute value on the abscissa.

3. Calculate the cumulative probability in the same way as step (2) for $A E$ index, but value shows the probability of the maximum $A E$ index greater than a given value.

4. The 1 min geosynchronous magnetic field data available from GOES [Singer, 1996] are also projected to the mean field-aligned coordinate system [Takahashi et al., 1990; Zong et al., 2007], in which the parallel direction $p$ is determined by 20 min sliding averaged magnetic field, the azimuthal direction is parallel to the cross product of the $(p)$ and the spacecraft position vector, and the radial direction $(r)$ completes the triad. Then the magnetic field data are filtered with the $150 \mathrm{~s}$ to $600 \mathrm{~s}$ band-pass filter (ULF Pc5 wave frequency band), and we use the maximum value of the filtered magnetic field magnitude for the $12 \mathrm{~h}$ prior and $12 \mathrm{~h}$ after the zero epoch time for each event in the three categories (ICME, SIR, and Alfénic) to represent the amplitude of the ULF wave activity. Sanny et al. [2007] found a local time dependence of ULF wave power, which varies by at most 1 order of magnitude during strong magnetic storms. However, the variation pattern of the ULF wave power shows to be a two-cycle sinusoidal function, while the peaks of the two cycles ( $12 \mathrm{~h}$ interval) change by at most half an order (the wave amplitude change at most a quarter order). Thus, the maximum value of the ULF wave amplitude in a $12 \mathrm{~h}$ interval in our analysis well represents the strength of ULF wave activity at geosynchronous orbit.

\section{Results}

Figures 1-3 show the superposed epoch analysis for all the $B_{s}$ events $\left(B_{z}<-5 \mathrm{nT}\right.$ and $\left.t>1 \mathrm{~h}\right)$ related with ICMEs and SIRs in the period of 1995-2004 and related with Alfvénic intervals during 1998-2004 [Zhang and Moldwin, 2014; Zhang et al., 2014]. There are 227 events defined as ICME-type southward IMF events, 198 cases categorized as SIR-type, and 56 events identified as Alfvénic $B_{s}$ events. We do not show or discuss results for shock-related events since there are only a few events (11). Figures $1 \mathrm{a}, 2 \mathrm{a}$, and $3 \mathrm{a}$ present a decrease to or below $-5 \mathrm{nT}$ in IMF $B_{z}$ at zero epoch time, while the median duration of strong $B_{s}$ intervals for ICME-, SIR-, and Alfvénic-type $B_{s}$ events last for about $4 \mathrm{~h}, 3 \mathrm{~h}$, and $2 \mathrm{~h}$, respectively, until they cross the horizontal dashed line again. Figures $1 \mathrm{~b}, 2 \mathrm{~b}$, and $3 \mathrm{~b}$ show the magnetic field amplitude $\left(B_{t}\right)$. There is a weak jump of all the three quartiles of $B_{t}$ at the zero epoch time for all the different classes of structures. The profile of median $B_{t}$ shows a gradual increase from $7.31 \mathrm{nT}$ to $11.67 \mathrm{nT}$ before the zero epoch time and then a decrease to $8.31 \mathrm{nT}$ until the end of the 2 day profile for ICME-type $B_{s}$ events. The median value of total magnetic field increases by $100 \%$ from $5.59 \mathrm{nT}$ during the first $24 \mathrm{~h}$ of the profile and decreases to $7.4 \mathrm{nT}$ for SIR-related $B_{s}$ events. Figures $1 C, 2 c$, and $3 c$ show that the average change of solar wind speed is insignificant for ICME-type and Alfvénic $B_{s}$ events but there are higher values for ICME events. For SIR-type $B_{s}$ events, there is a smooth increase from $350 \mathrm{~km} / \mathrm{s}$ to about $470 \mathrm{~km} / \mathrm{s}$ for the median values in $V_{\text {sw }}$ throughout the 2 days. The proton density in Figures $1 \mathrm{~d}, 2 \mathrm{~d}$, and $3 \mathrm{~d}$ indicates an increase after the zero epoch followed by a decrease for all $B_{s}$ events except for the Alfvénic ones. The increase of $N_{p}$ starts about $4 \mathrm{~h} / 8 \mathrm{~h}$ before and ends at the zero epoch for ICME-/SIR-related $B_{s}$ intervals. Some cases, especially from the ICME $B_{s}$ event list, show large deviations from the main band of the statistical distribution at some epoch time points. This could be caused by the different timescales of these events, and/or different amplitudes of the variations during these intervals, and/or different background solar wind/IMF conditions. However, the 


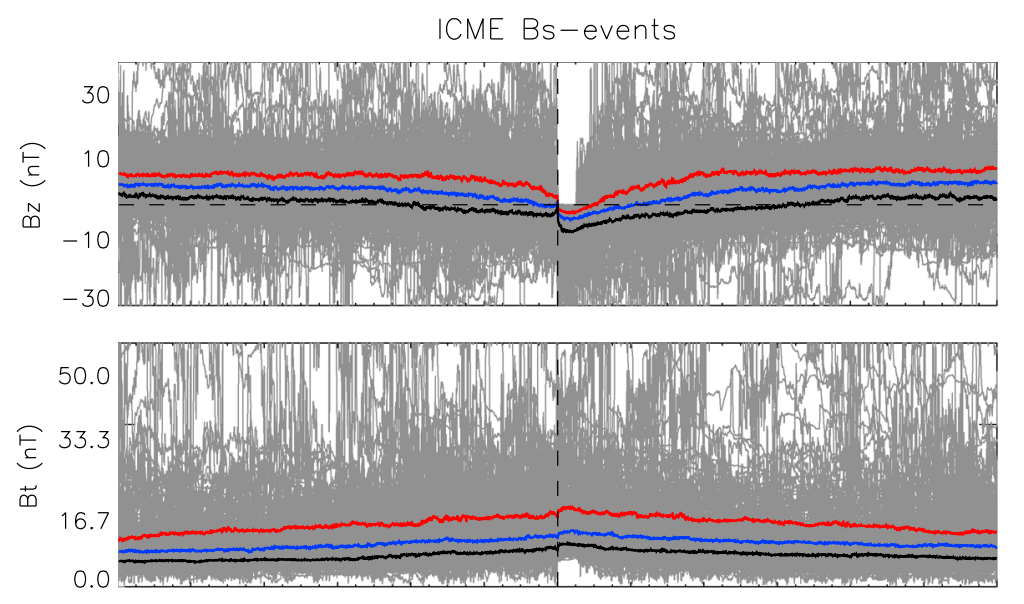

(A)

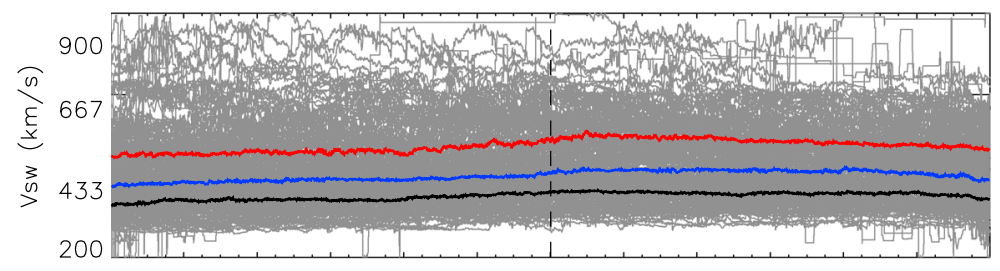

(B)

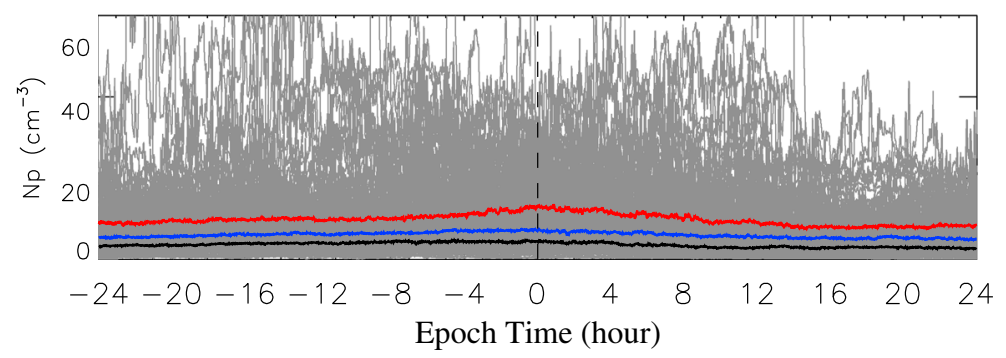

(C)

Figure 1. Superposed epoch analysis for all the 227 ICME-related $B_{s}$ intervals $\left(B_{z}<-5 \mathrm{nT}\right.$ and $\left.t>1 \mathrm{~h}\right)$ during 1995-2004 identified by Zhang and Moldwin [2014] and Zhang et al. [2014]. The epoch zero is set at the change of IMF $B_{z}$ drops under $-5 \mathrm{nT}$ denoted by the dashed vertical line, and the duration is $48 \mathrm{~h}$. The grey/red/blue/black are the temporal files of all the cases/upper/middle/lower quartile of (a) IMF $B_{z}$ (b) IMF amplitude, (c) solar wind speed $\left(V_{\text {sw }}\right)$, and (d) proton density $\left(N_{p}\right)$. The horizontal dashed line marks the threshold as $B_{z}=-5 \mathrm{nT}$. All the data are measured by the magnetometer on board Wind satellite and downloaded from Coordinated Data Analysis Web (CDAWeb, http://cdaweb. gsfc.nasa.gov/cgi-bin/eval1.cgi).

trend of the change of these parameters can be distinguished, which is the crucial result for studying the potential precursors of these strong southward IMF intervals.

Though the goal of this study is to demonstrate that IMF $B_{z}$ intervals often are proceeded by systematic variations in the solar wind and IMF parameters, not to develop a forecasting model, we did a preliminary study to test the predictability of the strong southward IMF intervals using the precursor signatures. We created a set of precursor signatures by using the mean precursor signature of all events (CIR, ICME, etc.) combined. We defined the precursor intervals as follows: $N_{p}$ shows a peak greater than $5 \mathrm{~cm}^{-3}$ at time $t_{0}$, the mean value of $B_{t}$ and $V_{s w}$ in the interval $t_{0}$ to $t_{0}+\mathrm{d} t$ is greater than that in $t_{0}-\mathrm{d} t$ to $t_{0}$, where $\mathrm{d} t=30 \mathrm{~min}$, the maximum value of $B_{t}$ and $V_{\mathrm{sw}}$ in this $1 \mathrm{~h}$ interval is greater than $5 \mathrm{nT}$ and $400 \mathrm{~km} / \mathrm{s}$ respectively, and the mean value of $B_{z}$ is less than $0 \mathrm{nT}$ for $t_{0}$ to $t_{0}+\mathrm{d} t$, from ACE upstream solar wind/IMF data for year 2005-2010, and found that $\sim 20 \%-60 \%$ (varies with year) of these precursors were followed by a strong IMF $B_{s}$ event in the following $12 \mathrm{~h}$. Note that we used Wind data to do the original analysis of the solar wind/IMF behavior prior to the $B_{s}$ events, while this preliminary analysis used ACE data. Also note that we combined all of the solar wind transients together in this preliminary analysis.

Figure 4 presents the cumulative probability distribution function for the SYM- $H$ index, which gives the probability that $S Y M-H$ will be smaller than the ordinate. The curves are based on data recorded during $12 \mathrm{~h}$ 

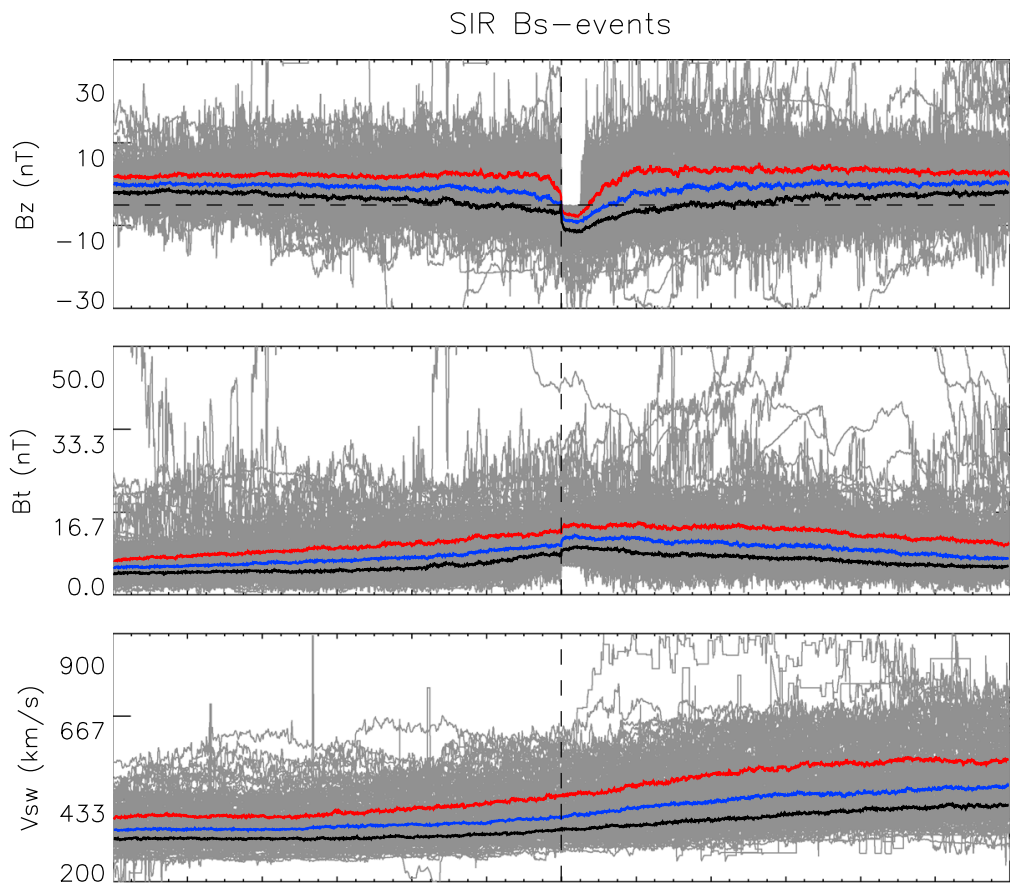

(C)

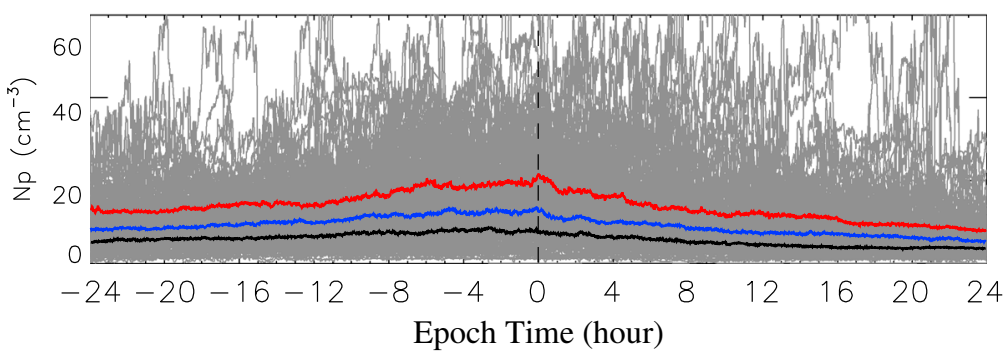

Figure 2. The same format as Figure 1 for all the 198 SIR-related $B_{S}$ intervals.

before (more northward) and after (when $B_{z}>-5 \mathrm{nT}$ or more "northward") the zero epoch illustrated in Figures $1-3$. SYM- $H$ at value of $-50 \mathrm{nT}$ is marked by the vertical line, which is widely used as the threshold of a geomagnetic storm. For all types of $B_{s}$ intervals except Alfvénic events, the probability that SYM- $H$ will be below a given value is greater after the zero epoch than before the zero epoch. Within either $12 \mathrm{~h}$ interval, high $V_{\text {sw }}$ conditions are more geoeffective than slow $V_{\text {sw }}$ conditions for ICME- and SIR-related events. However, for Alfvénic $B_{s}$ intervals, the fast solar wind before the zero epoch leads to higher probability of geomagnetic storms than intervals with slow solar wind after the zero epoch. The data show that fast solar wind intensifies geomagnetic storms, while the strong southward IMF is the key for triggering such activity, except for Alfvénic events.

Figure 5 shows the difference between high and low proton density in terms of the probability to trigger geomagnetic storms in the same format as Figure 4. For the ICME-type $B_{s}$ intervals, the cumulative probability function for low-density conditions is above the high-density conditions when minimum SYM- $H$ is less negative than $-60 \mathrm{nT}$ during either of the $12 \mathrm{~h}$ windows. For the $B_{s}$ intervals related with $\mathrm{SIR}$, the low proton density condition is more likely to trigger a stronger geomagnetic storm during the whole $24 \mathrm{~h}$ period, which is the same for Alfvénic $B_{s}$ events (though the PDF are very similar). However, like the effect of $V_{s w}$, the probability function of low proton density before the zero epoch overlaps with the high-density conditions after the zero epoch for Alfvénic events. This indicates that solar wind density does not intensify geomagnetic activity significantly or correlate with SYM- $H$ well, suggesting that it impacts the magnetosphere differently than solar wind speed.

The comparison of $A E$ index distribution functions between conditions of fast and slow solar wind during the $12 \mathrm{~h}$ intervals before and after the turning of IMF to more southward is illustrated in Figure 6 . The format 

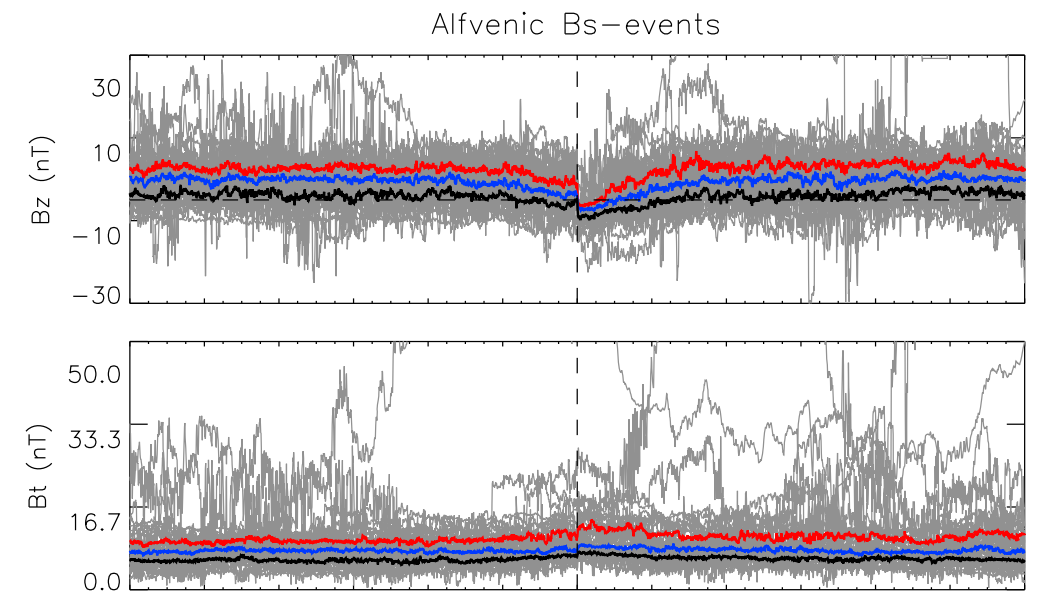

(B)

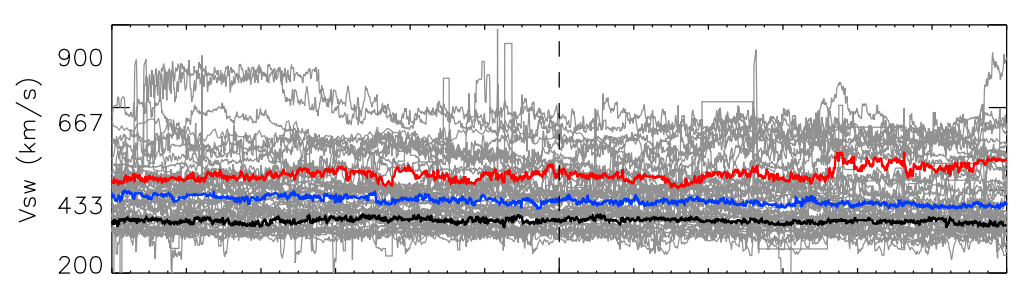

(C)

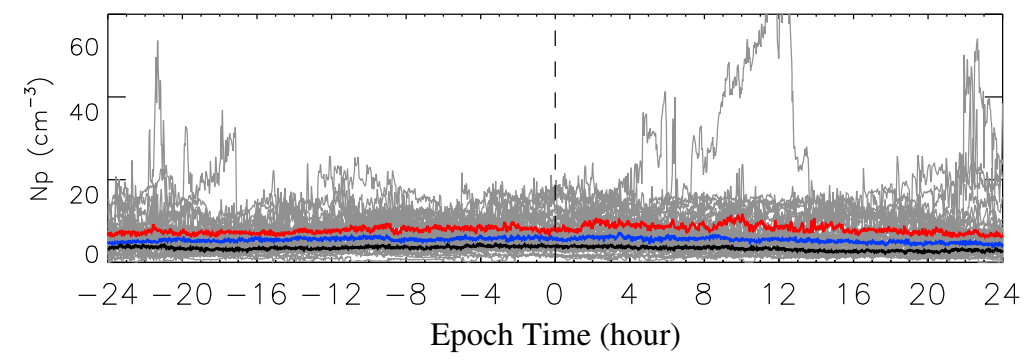

(D)

Figure 3. The same format as Figure 1 for all the 56 Alfvénic $B_{s}$ intervals in the period of 1998-2004.
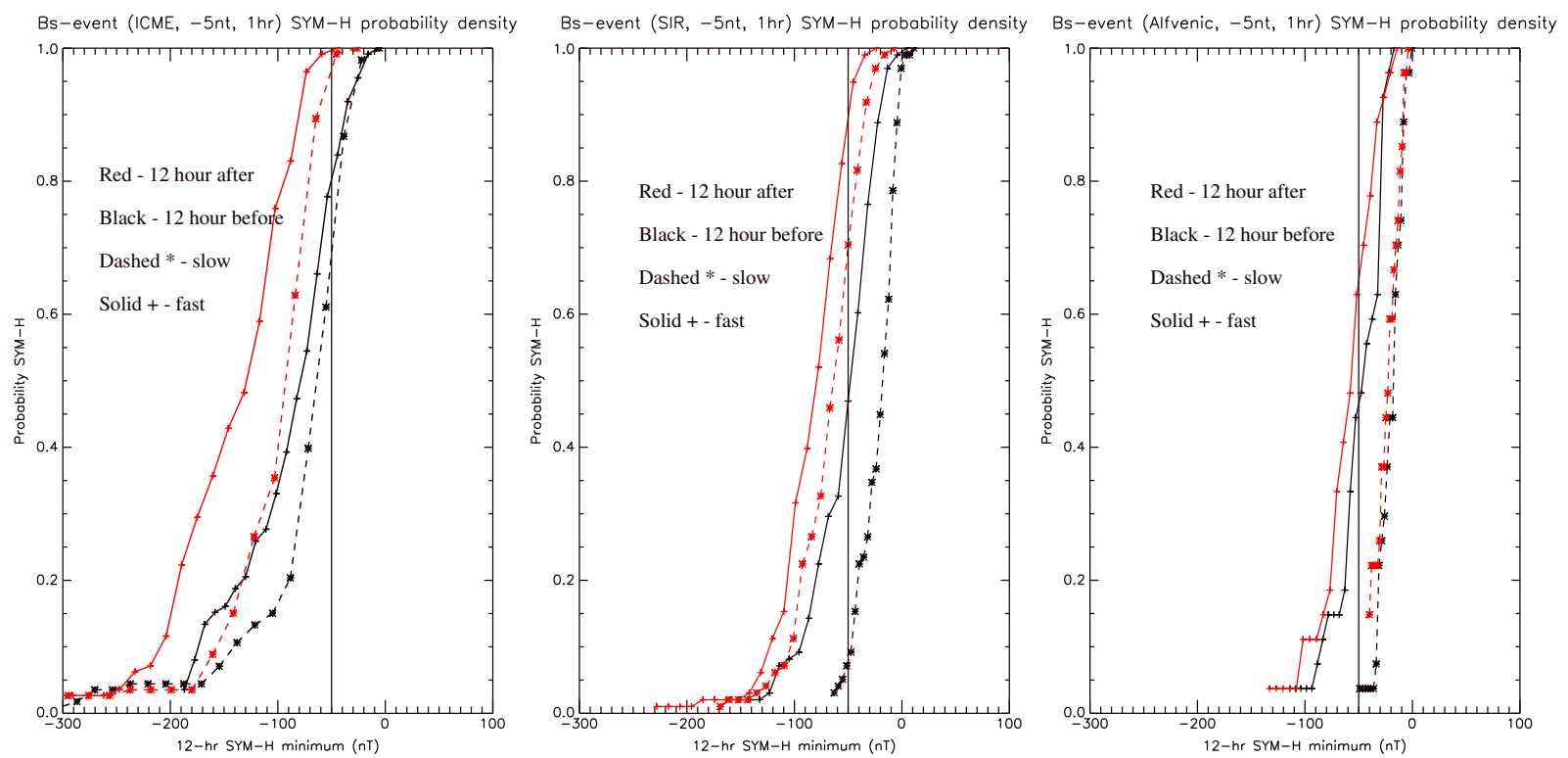

Figure 4. Probabilistic curves for SYM-H for $12 \mathrm{~h}$ before (black) and after (red) the zero epoch of southward IMF intervals associated with different solar wind structures. Each case shows two curves corresponding to high and low solar wind speed. The solid/dashed line is for events in which the solar wind speed is faster/slower than the median speed for all cases. The vertical line marks the SYM- $H$ value of $-50 \mathrm{nT}$. 

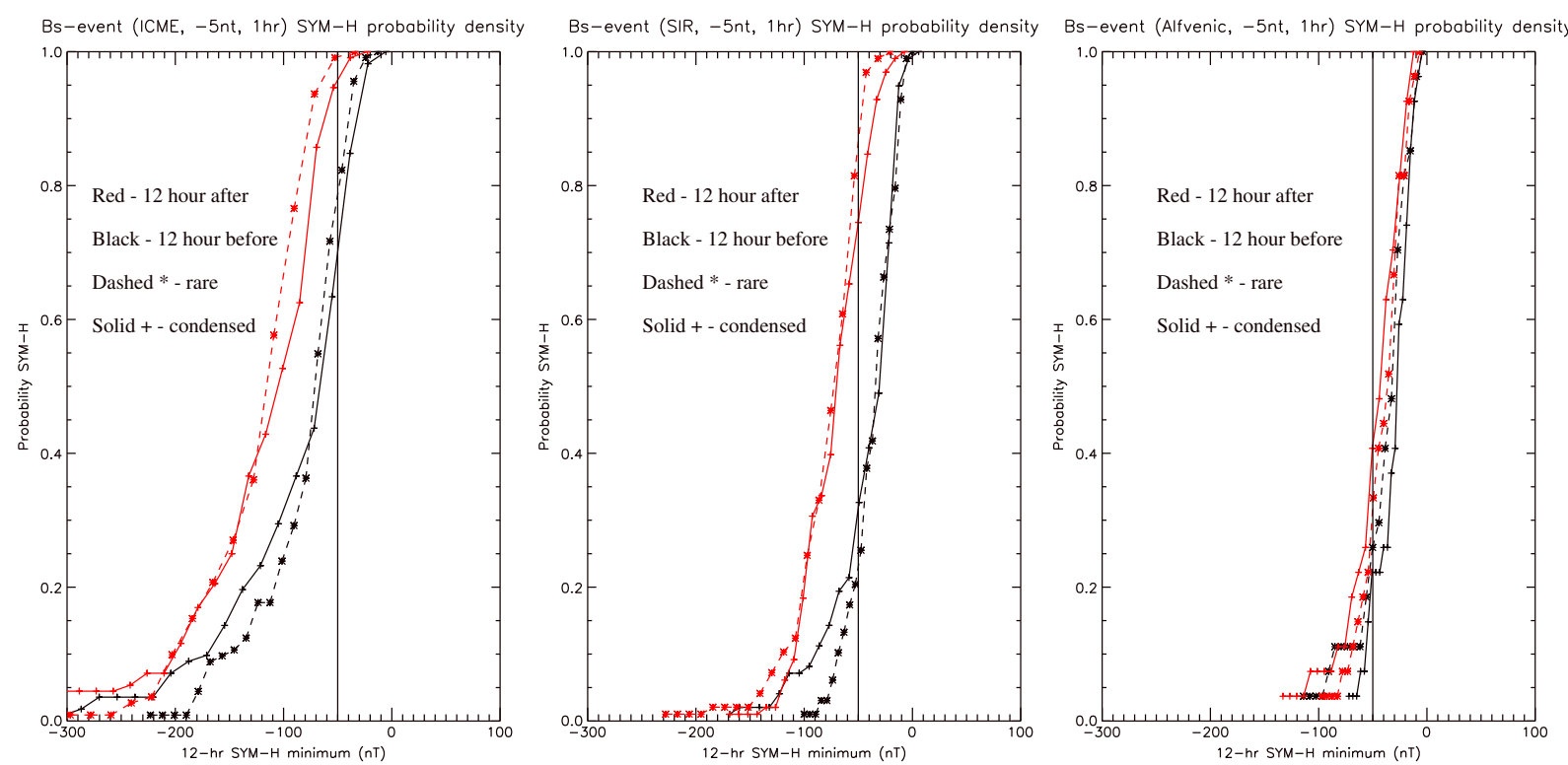

Figure 5. The same format as Figure 4 but showing the impact of high or low proton density.

is the same as Figure 4, but the function shows the probability of $A E$ exceeding the ordinate. $A E$ at value of $1000 \mathrm{nT}$ is marked by the vertical line, which is often taken as the threshold of a geomagnetic substorm. It shows that the distribution function for fast solar wind always has a greater $A E$ than slow solar wind during both $12 \mathrm{~h}$ intervals for all kinds of $B_{s}$ events, which is the same as the SYM-H. However, fast solar wind during the $12 \mathrm{~h}$ interval before the zero epoch time presents higher occurrence probability of larger $A E$ than the slow solar wind during the $12 \mathrm{~h}$ interval after that for all types of $B_{s}$ intervals, except that the two curves almost overlap for SIR-type $B_{s}$ events. It is suggested that fast solar wind is important in inducing and amplifying geomagnetic substorms for most of the cases, but strong southward IMF is not necessarily the key.

We also performed analysis of ultralow-frequency (ULF) wave amplitude at geosynchronous orbit using GOES data. Figure 7 illustrates the cumulative probability of the maximum amplitude of the magnetic
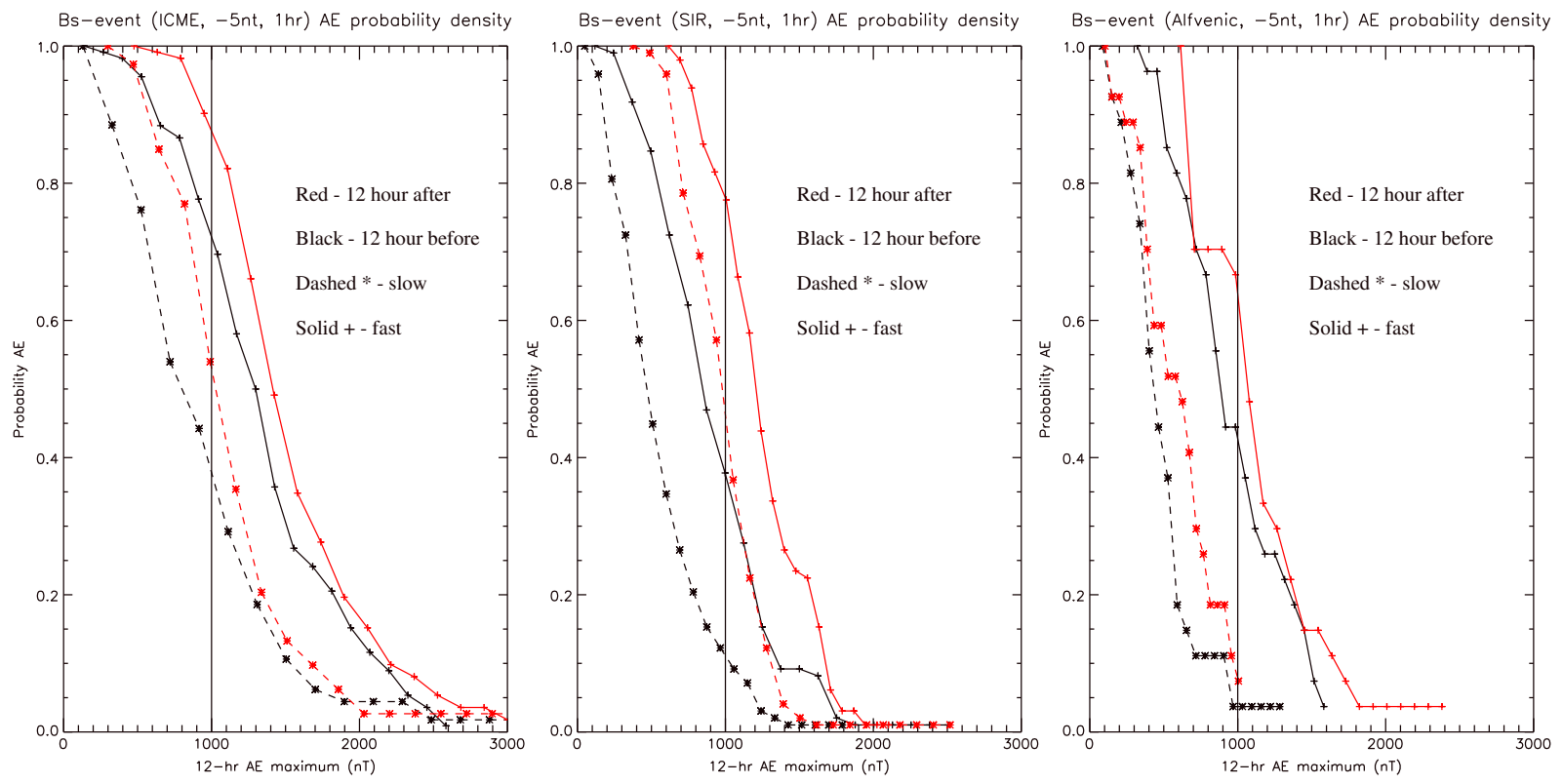

Figure 6. The same format as Figure 4 but for cumulative probability distribution of $A E$ index. 

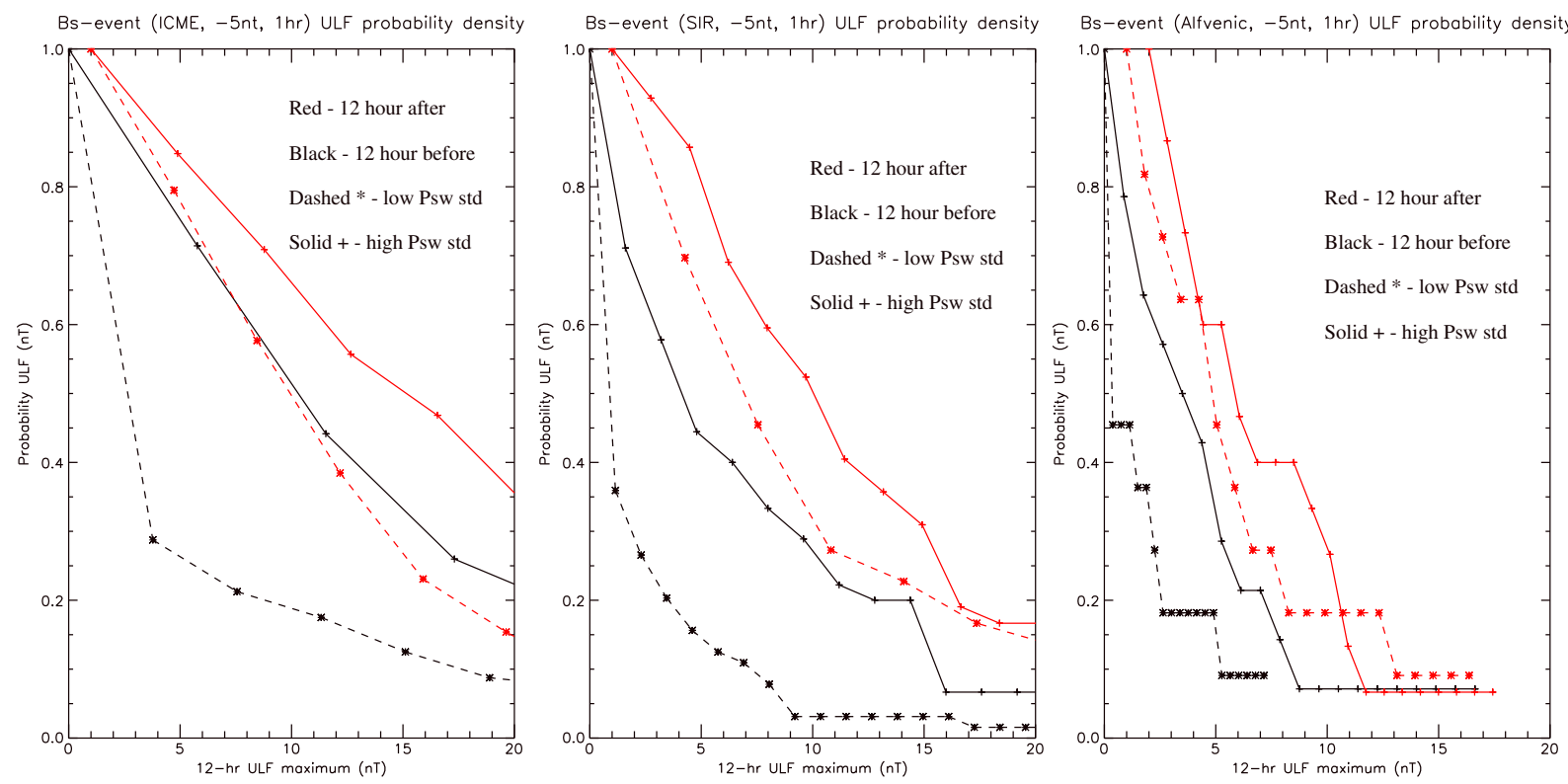

Figure 7. The same format as Figure 4 but for cumulative probability distribution of the maximum value of total magnetic field in ULF wave frequency band $(1.6-6.7 \mathrm{mHz})$ measured by GOES satellite, in terms of the standard deviation of solar wind dynamic pressure $\left(P_{\mathrm{sw}}\right)$.

field in ULF wave frequency band $\left(1.6-6.7 \mathrm{mHz}\right.$ ) for different degrees of $P_{\mathrm{sw}}$ variability, in the same format as Figure 4. It shows that for all types of $B_{s}$ events, the higher variability of $P_{s w}$ is related with larger ULF wave amplitude in both $12 \mathrm{~h}$ windows. For the SIR-type and Alfvénic $B_{s}$ events, the larger perturbation of $P_{\mathrm{sw}}$ during the more northward interval ( $12 \mathrm{~h}$ before) has lower probability to induce more intense ULF wave activity than the weaker perturbation of $P_{\text {sw }}$ during the more southward interval ( $12 \mathrm{~h}$ after). But for the ICME-related $B_{s}$ events, larger deviation of $P_{\text {sW }}$ during the more northward IMF period is even more associated with stronger perturbations of ULF waves in the magnetosphere than lower $P_{\text {sw }}$ deviations during the more southward IMF interval. We also tested the relationship between the median value of $P_{\mathrm{sW}}$ and ULF wave activity and obtained similar results. However, the probability of high ULF wave amplitudes are enhanced for weak $P_{\text {sw }}$ compared to strong $P_{\text {sw }}$ during the $12 \mathrm{~h}$ after the zero epoch time for SIR-type $B_{s}$ events. These results suggest that the variability of solar wind dynamic pressure (rather than absolute value) plays the most significant role in inducing intense ULF wave activity in Earth's magnetosphere; however, the strong southward IMF associated with solar wind transients is more likely to trigger geomagnetic storms as well as important in intensifying ULF wave amplitude in this frequency band.

\section{Discussions and Conclusions}

This paper is a follow-on study of McPherron and Siscoe [2004] expanding the solar wind marker to all the geoeffective solar wind transients that have been studied previously [Denton et al., 2006; Tsurutani et al., 2006, 2013]. Instead of east-west flow deflection change of CIR, we focus on the southward component of the interplanetary magnetic field (southward IMF), which is accepted as the most important trigger for geomagnetic disturbances [Dungey, 1961; Fairfield and Cahill, 1966; Arnoldy, 1971; Akasofu, 1979]. We related those southward IMF intervals $\left(B_{z}<-5 \mathrm{nT}\right.$ and $\left.t>1 \mathrm{~h}\right)$ with different kinds of solar wind phenomena and obtain the statistical properties of other solar wind/IMF parameters. For the analysis of probability distribution function of geomagnetic indices, we also include several solar wind parameters and geomagnetic activity indices.

We found that there is a distinct set of solar wind/IMF parameter profiles for southward IMF intervals related with different types of solar wind transients. Our study showed that statistically, there is a significant peak of magnetic field amplitude close to the start time of these intervals for ICME- and SIR-type southward IMF events and that the median solar wind speed experiences a smooth but significant increase during the 2 day profile around the SIR-type $B_{s}$ events as expected. We also found that the proton density changes significantly during the intervals related with ICMEs and SIRs in this study while their time scales are 
different. We also calculated the east-west electric field (not presented here) as $E_{y}=V_{x} \times B_{z}-V_{z} \times B_{x}$ where $x$ is positive toward the Sun, $z$ is positive northward, and thus $y$ is positive duskward and found that $E_{y}$ increases following the zero epoch for ICME- and SIR-related cases, decreases to values even less than the quiet condition, and then increases again for the ICME and SIR events. The preliminary work to test the predictability of southward IMF intervals shows that $20 \%-60 \%$ of the precursors are followed by IMF $B_{s}$ events and $20 \%$ of $B_{s}$ events have precursors, but both rates may be higher if the intervals examined are classified into different solar wind transient types (CIR, ICME, etc.) since the background conditions and trend of changes in solar wind/IMF parameters are not very similar among them. This preliminary study created a precursor profile by averaging the solar wind/IMF signatures for all IMF $B_{s}$ events, not distinguishing between the different solar wind transient types. This can be done for CIR and ICME based on solar observations.

The results from the probabilistic forecasting analysis of geomagnetic activity indices show that solar wind parameters affect the Earth's magnetosphere in different ways; also, different regions of geomagnetic field respond to the same solar wind parameters in different ways. Like weather forecasts, this technique provides a tool for predicting the occurrence rate of geomagnetic activity with intensity level based on a combination of various solar wind quantities, obtainable from either measurements or models. This study contributes to our understanding of IMF $B_{z}$ and is a bridge for space weather forecasting, while space weather simulations develop better methods of modeling southward IMF events.

Currently, solar/heliospheric models either do not simulate the IMF $B_{z}$ component or provide low-accuracy forecasts; however, they provide good prediction of solar wind velocity, density, and magnetic field amplitude for large-scale solar wind structures [Tóth et al., 2007; Parsons, 2011]. Based on this study, we propose that some strong southward IMF events are predictable. Furthermore, the probabilistic analysis of geomagnetic activity indices, under specific sets of solar wind conditions, provides a tool to make better use of available observations and models for space weather forecasting.

Our next step is to analyze more measurements, such as temperature, electric currents, EUV, X-ray, and white-light images, which could provide observations of solar transients for up to several days in advance, and also perform derivation of other parameters, like Alfvén wave energy density, from available in situ measurements, around the southward IMF events. Considering the distinctive formation mechanisms for each solar wind transient and the strong embedded southward IMF, it is possible to obtain a unique set of signatures that could be measured several days or hours in advance or be predicted from current models. Thus, probabilistic forecasting the occurrence of long-duration, large-amplitude southward IMF intervals during solar wind transients may be possible.

\section{References}

Akasofu, S. I. (1979), A search for the interplanetary quantity controlling the development of geomagnetic storms, Q. J. R. Astron. Soc., 20, 119-137.

Arnoldy, R. L. (1971), Signature in the interplanetary medium for substorms, J. Geophys. Res., 76, 5189-5201.

Denton, M. H., J. E. Borovsky, R. M. Skoug, M. F. Thomsen, B. Lavraud, M. G. Henderson, R. L. McPherron, J. C. Zhang, and M. W. Liemohn (2006), Geomagnetic storms driven by ICME- and CIR-dominated solar wind, J. Geophys. Res., 111, A07S07, doi:10.1029/2005JA011436. Dungey, J. W. (1961), Interplanetary magnetic field and the auroral zones, Phys. Rev. Lett., 6, 47-48.

Echer, E., and W. D. Gonzalez (2004), Geoeffectiveness of interplanetary shocks, magnetic clouds, sector boundary crossings and their combined occurrence, Geophys. Res. Lett., 31, L09808, doi:10.1029/2003GL019199.

Fairfield, D. H., and J. L. J. Cahill (1966), Transition region magnetic field and polar magnetic disturbances, J. Geophys. Res., 71(1), 155-169.

Green, J. C., T. G. Onsager, T. P. O'Brien, and D. N. Baker (2004), Testing loss mechanisms capable of rapidly depleting relativistic electron flux in the Earth's outer radiation belt, J. Geophys. Res., 109, A12211, doi:10.1029/2004JA010579.

Jian, L. K., C. Russell, J. G. Luhmann, and R. M. Skoug (2006a), Properties of stream interactions at one AU during 1995-2004, Sol. Phys., $239,337-392$.

Jin, M., et al. (2012), A global two-temperature corona and inner heliosphere model: A comprehensive validation study, Astrophys. J., $745,6$.

Liou, K., C.-C. Wu, D. Murray, S.-T. Wu, N. Rich, S. Plunkett, L. Simpson, C. D. Fry, and K. Schenk (2014), Global simulation of extremely fast coronal mass ejection on 23 July 2012, J. Atmos. Sol. Terr. Phys., 121, 32-41.

Lopez, R. E., M. Wiltberger, S. Hernandez, and J. G. Lyon (2004), Solar wind density control of energy transfer to the magnetosphere, Geophys. Res. Lett., 31, L08804, doi:10.1029/2003GL018780.

McPherron, R. L., and G. Siscoe (2004), Probabilistic forecasting of geomagnetic indices using solar wind air mass analysis, Space Weather, 2, S01001, doi:10.1029/2003SW000003.

Newell, P. T., T. Sotirelis, K. Liou, C.-I. Meng, and F. J. Rich (2007), A nearly universal solar wind-magnetosphere coupling function inferred from 10 magnetospheric state variables, J. Geophys. Res., 112, A01206, doi:10.1029/2006JA012015.

O'Brien, T. P., R. L. McPherron, and D. Sornette (2001), Which magnetic storms produce relativistic electrons at geosynchronous orbit?, J. Geophys. Res., 106, 15,533-15,544. 
Owens, M. J., T. S. Horbury, R. T. Wicks, S. L. McGregor, N. P. Savani, and M. Xiong (2014), Ensemble downscaling in coupled solar wind-magnetosphere modeling for space weather forecasting, Space Weather, 12, 395-405, doi:10.1002/2014SW001064.

Palmroth, M., T. I. Pulkkinen, P. Janhunen, and C.-C. Wu (2003), Stormtime energy transfer in global MHD simulation, J. Geophys. Res., 108(A1), 1048, doi:10.1029/2002JA009446.

Parsons, A. (2011), Wang-Sheeley-Arge-Enlil cone model transitions to operations, Space Weather, 9, S03004, doi:10.1029/2011SW000663. Sanny, J., J. A. Tapia, D. G. Sibeck, and M. B. Moldwin (2002), Quiet time variability of the geosynchronous magnetic field and its response to the solar wind, J. Geophys. Res., 107(A12), 1443, doi:10.1029/2002JA009448.

Sanny, J., D. Judnick, M. B. Moldwin, D. Berube, and D. G. Sibeck (2007), Global profiles of compressional ultralow frequency wave power at geosynchronous orbit and their response to the solar wind, J. Geophys. Res., 112, A05224, doi:10.1029/2006JA012046.

Shen, F., C. Shen, J. Zhang, P. Hess, Y. Wang, X. Feng, H. Cheng, and Y. Yang (2014), Evolution of the 12 July 2012 CME from the Sun to the Earth: Data-constrained three-dimensional MHD simulations, J. Geophys. Res. Space Physics, 119, 7128-7141, doi:10.1002/2014JA020365.

Singer, H. J. (1996), Monitoring space weather with the GOES magnetometers, Proc. SPIE, 2812, 299-308.

Skoug, R. M., J. T. Gosling, J. T. Steinberg, D. J. McComas, C. W. Smith, N. F. Ness, Q. Hu, and L. F. Burlaga (2004), Extremely high speed solar wind: 29-30 October 2003, J. Geophys. Res., 109, A09102, doi:10.1029/2004JA010494.

Tóth, G., D. L. D. Zeeuw, T. I. Gombosi, W. B. Manchester, A. J. Ridley, I. V. Sokolov, and I. I. Roussev (2007), Sun-to-thermosphere simulation of the 28-30 October 2003 storm with the Space Weather Modeling Framework, Space Weather, 5, S06003, doi:10.1029/2006SW000272.

Takahashi, K., and A. Y. Ukhorskiy (2008), Timing analysis of the relationship between solar wind parameters and geosynchronous Pc5 amplitude, J. Geophys. Res., 113, A12204, doi:10.1029/2008JA013327.

Takahashi, K., R. W. McEntire, A. T. Y. Lui, and T. A. Potemra (1990), lon flux oscillations associated with a radially polarized transverse Pc5 magnetic pulsation, J. Geophys. Res., 95, 3717-3731.

Tsurutani, B. T., and W. D. Gonzalez (1987), The cause of High Intensity Long-Duration Continuous AE Activity (HILDCAAs): Interplanetary Alfvén wave trains, Planet. Space Sci., 35, 405-412.

Tsurutani, B. T., et al. (2006), Corotating solar wind streams and recurrent geomagnetic activity: A review, J. Geophys. Res., 11, A07S01, doi:10.1029/2005JA011273.

Tsurutani, B. T., W. D. Gonzalez, Y. Kamide, and J. K. Arballo (2013), The Interplanetary causes of magnetic storms: A review, in Magnetic Storms, edited by B. T. Tsurutani et al., pp. 77-89, AGU, Washington, D. C.

Zhang, X.-Y., and M. B. Moldwin (2014), The source, statistical properties, and geoeffectiveness of long-duration southward interplanetary magnetic field intervals, J. Geophys. Res. Space Physics, 119, 658-669, doi:10.1002/2013JA018937.

Zhang, X.-Y., M. B. Moldwin, J. T. Steinberg, and R. M. Skoug (2014), Alfvén waves as a possible source of long-duration, large-amplitude, and geoeffective southward IMF, J. Geophys. Res. Space Physics, 119, 3259-3266, doi:10.1002/2013JA019623.

Zong, Q.-G., et al. (2007), Ultralow frequency modulation of energetic particles in the dayside magnetosphere, Geophys. Res. Lett., 34, L12105, doi:10.1029/2007GL029915.

Zurbuchen, T. H., and I. G. Richardson (2006), In-situ solar wind and magnetic field signature of interplanetary coronal mass ejections, Space Sci. Rev., 123, 31-43. 